\title{
Sobre foliaciones y formas diferenciables
}

\author{
Liliana Olga Jurado Cerrón ${ }^{1}$ y Galindo Taza Chamb $2^{2}$
}

Resumen: En este trabajo pretendemos estudiar las foliaciones de codimensión arbitraria relacionados con las formas diferenciables, teniendo dos resultados principales. El primer resultado prueba que a partir de una foliación de codimensión $q$ en $\mathbb{C}^{n}$ obtenemos una foliación en $\mathbb{C P}^{n}$. El segundo resultado demuestra la caracterización de las foliaciones afines transversalmente de codimensión arbitraria en términos de matrices.

Palabras clave: foliaciones; formas diferenciables; matrices.

\section{About foliations and differential forms}

\begin{abstract}
In this work we aim to study the foliations of arbitrary codimension related to the differential forms, obtaining two main results. In the first result we showed that from a foliation of codimension $q$ in $\mathbb{C}^{n}$ one can obtain a foliation in $\mathbb{C P}^{n}$. The second result shows the characterization of the transversely affine foliations of arbitrary codimension in terms of matrices.
\end{abstract}

Keywords: Foliations; differential forms; matrices.

Recibido: 10/11/2019. Aceptado: 05/08/2020. Publicado online: 18/08/2020.

(C) Los autores. Este artículo es publicado por la Revista PESQUIMAT de la Facultad de Ciencias Matemáticas, Universidad Nacional Mayor de San Marcos. Este es un artículo de acceso abierto, distribuido bajo los términos de la licencia Creative Commons Atribucion-No Comercia-CompartirIgual 4.0 Internacional.(http://creativecommons.org/licenses/by-nc-sa/4.0/) que permite el uso no comercial, distribución y reproducción en cualquier medio, siempre que la obra original sea debidamente citada. Para uso comercial, por favor póngase en contacto con revistapesquimat.matematica@unmsm.edu.pe

\footnotetext{
${ }^{1}$ PUCP, Departamento Académico de Ciencias, Sección Matemáticas. e-mail: ljuradoc@pucp.edu.pe

${ }^{2}$ UNMSM, Facultad de Ciencias Matemáticas, e-mail: gtazac@unmsm.edu.pe
} 


\section{Introducción}

La teoría de las foliaciones es una de las áreas de la Matemática que realiza la confluencia de varios dominios distintos: topología, sistemas dinámicos, topología diferencial, geometría, entre otros. Su grado de desenvolvimiento permitió una mejor comprensión de varios fenómenos de naturaleza matemática y de naturaleza físico matemático, contribuyendo de forma importante para el desenvolvimiento de las diversas áreas arriba citadas, y que en el inicio la abastecieron con la teoría. Hoy teoremas clásicos como los teoremas de estabilidad de Reeb, teorema de existencia de holonomía no trivial de Haefliger, y de existencia de una hoja compacta de S. Novikov, y estudio de foliaciones con crecimiento subexponencial desarrollado por J. Plante hoy son buscados para situaciones más generales: foliaciones con singularidades, foliaciones holomorfas, etc. Matemáticos renombrados comienzan a investigar tales fenómenos (por ejemplo E. Ghys, M. Brunella, C. Camacho, D. Cerveau, A. Lins Neto, P. Sad, Bruno Scárdua entre otros).

El sistema de ecuaciones diferenciales de la forma

$$
\frac{d y}{d x}=\frac{P(x, y)}{Q(x, y)}
$$

donde $P$ y $Q$ son polinomios de dos variables complejas, se dice que una función es una integral primera para el sistema (1), si es constante a lo largo de las soluciones de (1), donde por la Definición 2.1, las soluciones son hojas de una foliación de dimensión uno en $\mathbb{C}^{2}$.

En [8], el autor M. Singer caracteriza el sistema (1) que posee una integral primera que esta relacionada con la estructura transversalmente afin de la foliación. Motivado por [8], el autor B. Scárdua en [7] extiende los resultados para foliaciones de dimensión uno y codimensión uno en $\mathbb{C}^{n}$.

El objetivo de este trabajo es proveer los recursos necesarios para la investigación de las foliaciones de codimensión arbitraria, extenderemos así algunas propiedades de foliaciones de codimensión uno en A. Lins Neto ([5], pág. 72) y algunos ejemplos para foliaciones de codimensión arbitraria y mostraremos una caracterización del caso de foliaciones transversalmente afines en términos de matrices.

\section{Foliaciones y formas diferenciables}

Definición 2.1 Una foliación holomorfa $\mathcal{F}$, de codimensión $q$, en una variedad compleja $M$, de dimensión $m$, donde $1 \leq m-q \leq m$, es una descomposición de $M$ en sub-variedades holomorfas inmersas conexas de dimensión $m-q$, llamadas hojas de $\mathcal{F}$, con las propiedades siguientes:

1. Para todo punto $p \in M$ existe una única hoja $L_{p}$ de $\mathcal{F}$ pasando por $p$. Si $q \in L_{p}$ entonces $L_{p}=L_{q}$.

2. Para todo $p \in M$, existe una carta local holomorfa $(U, \phi)$ de $M$ con $p \in U$, y tal que $\phi: U \rightarrow V_{m-q} \times V_{q}$, donde $V_{m-q}$ y $V_{q}$ son abiertos conexos de $\mathbb{C}^{m-q}$ y $\mathbb{C}^{q}$ respectivamente. Para todo $(x, y) \in V_{m-q} \times V_{q}$ la sub-variedad de dimensión $m-q$ de $U, \phi^{-1}\left(V_{m-q} \times\{y\}\right)$, es un abierto de $L_{q}$, donde $q=\phi^{-1}(x, y)$.

En seguida veremos una de las maneras de definir una foliación, equivalente a la anterior, y que será usado a lo largo del trabajo. La demostración de dicha equivalencia puede ser encontrada en A. Lins Neto - B. Scárdua ([5], pág. 13-16). 
Proposición 2.1 Sea $\mathcal{F}$ una foliación de codimensión $k$ de $M$, como en la Definición 2.1, entonces $\mathcal{F}$ es dado por un cubrimiento abierto $M=\cup_{\alpha \in A} U_{\alpha}$ y por colecciones $\left\{y_{\alpha}\right\}_{\alpha} y\left\{g_{\alpha \beta}\right\}_{U_{\alpha} \cap U_{\beta} \neq \emptyset}$, que satisfacen:

1. $\forall \alpha \in A, y_{\alpha}: U_{\alpha} \rightarrow \mathbb{C}^{k}$ es una submersión.

2. Si $U_{\alpha} \cap U_{\beta} \neq \emptyset$ entonces $y_{\alpha}=g_{\alpha \beta}\left(y_{\beta}\right)$ donde $g_{\alpha \beta}: y_{\beta}\left(U_{\alpha} \cap U_{\beta}\right) \subset \mathbb{C}^{k} \rightarrow y_{\alpha}\left(U_{\alpha} \cap U_{\beta}\right) \subset$ $\mathbb{C}^{k}$ es un difeomorfismo local holomorfo.

En este caso las placas de $\mathcal{F}$ en $U_{\alpha}$ son conjuntos de la forma $y_{\alpha}^{-1}(q), q \in V_{\alpha}$.

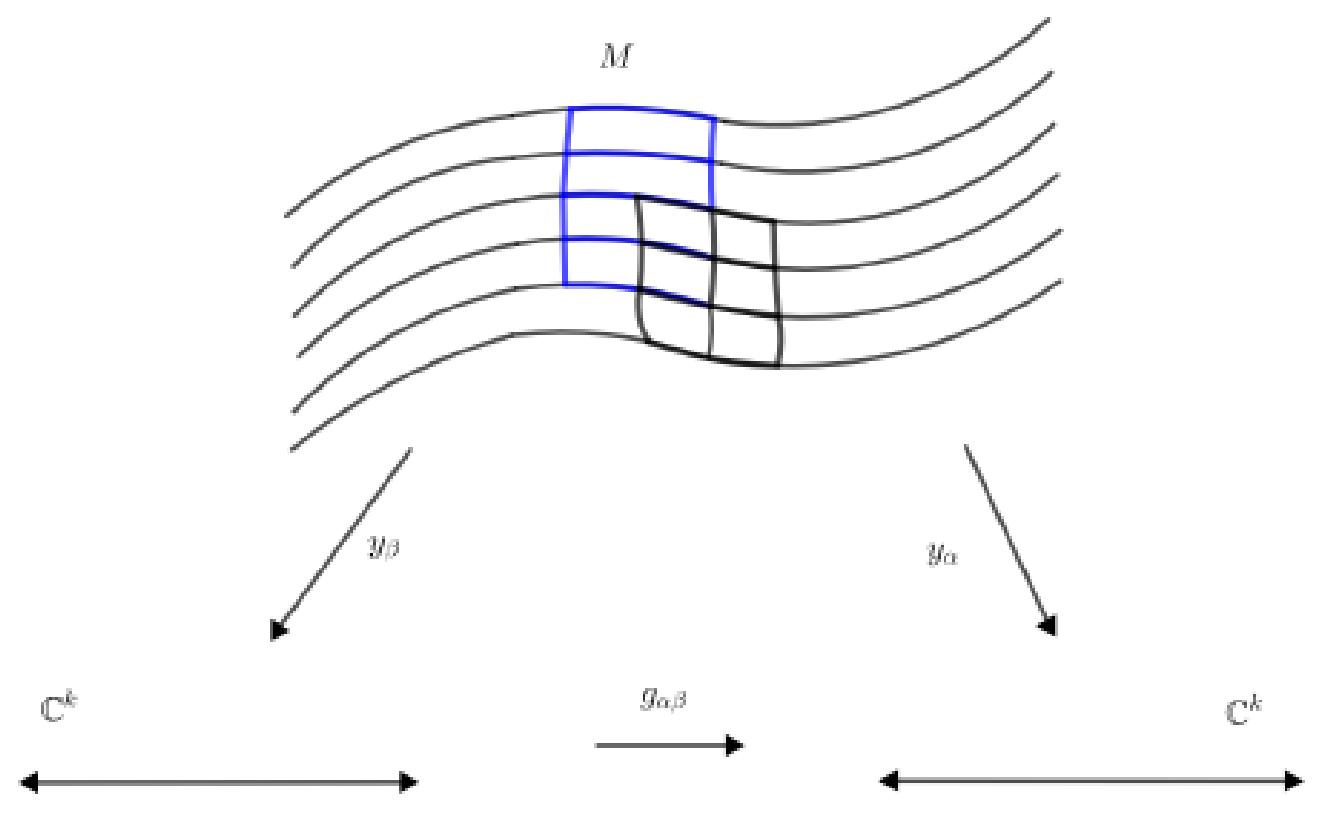

Figura 1: Aspecto local de una foliación de dimensión uno en una variedad de dimensión dos.

Nuestro interés es un tipo particular de foliaciones, que son las foliaciones transversalmente afines, veamos a seguir la definición.

Definición 2.2 Una foliación holomorfa de codimensión q sobre $M^{n}$ es llamada transversalmente afin, si existe una familia $\left\{Y_{i}: U_{i} \rightarrow \mathbb{C}^{q}\right\}_{i \in I}$ de submersiones holomorfas, definidas en conjuntos abiertos $U_{i} \subset M$, que definen $\mathcal{F}$ y que satisfacen $M=\cup_{i \in I} U_{i}$ y con relaciones afines $Y_{i}=A_{i j} Y_{j}+B_{i j}$ para algún $A_{i j}: U_{i} \cap U_{j} \rightarrow G L_{q}(\mathbb{C}), B_{i j}: U_{i} \cap U_{j} \rightarrow \mathbb{C}^{q}$ localmente constantes en cada $U_{i} \cap U_{j} \neq \emptyset$.

El teorema a seguir es una caracterización de una foliación transversalmente afín de codimensión uno con formas diferenciables, esencialmente dice que existe una forma cerrada tal que se relaciona con la forma que define la foliación, y viceversa, la demostración puede ser encontrada en A. Lins Neto - B. Scárdua (5], pág. 263-268).

Teorema 2.2 Sea $\mathcal{F}$ una foliación holomorfa de codimensión uno en una variedad compleja $M$. Suponga que $\mathcal{F}$ puede ser definida por una forma meromorfa, esto es, que existe una 1 -forma integrable meromorfa $\Omega$, que define $\mathcal{F}$ fuera de su divisor de polos, $(\Omega)_{\infty}$. La foliación $\mathcal{F}$ es transversalmente afín en el abierto $U=M \backslash$ sing $(\mathcal{F})$ si, y solamente si, existe una 1 -forma meromorfa $\eta$ en $M$ que satisface las siguientes propiedades:

1. $\eta$ es cerrada. 
2. $d \Omega=\eta \wedge \Omega$.

3. $\eta_{\infty}=\Omega_{\infty}$.

Además, dos pares $(\Omega, \eta)$ y $\left(\Omega^{\prime}, \eta^{\prime}\right)$ definen la misma estructura afín para $\mathcal{F}$ en $U$ si, y solamente si, existe una aplicación meromorfa $g: M \rightarrow \mathbb{C} \cup\{\infty\}$ que satisface $\Omega^{\prime}=g \Omega$ y $\eta^{\prime}=\eta+\frac{d g}{g}$ en $U$.

En este trabajo demostraremos el Teorema 2.2 en un sentido para foliaciones de dimensión arbitrária, para esto primeramente precisamos de las seguientes definiciones y proposiciones.

Definición 2.3 Dado el sistema $\Omega:=\left\{\Omega_{1}, \ldots, \Omega_{q}\right\}$, 1-formas holomorfas definidas en un conjunto abierto $U \subset M$, es integrable si para cada $j \in\{1, \ldots, q\}$ tenemos $d \Omega_{j} \wedge \Omega_{1} \wedge \ldots \wedge \Omega_{q}=0$ en $U$.

Proposición 2.3 Dado un sistema integrable $\Omega:=\left\{\Omega_{1}, \ldots, \Omega_{q}\right\}$, donde $\left\{\Omega_{i}\right\}_{i=1}^{q}$ son linealmente independientes sobre un abierto $U \subset M$, entonces por el Teorema de Frobenius existe una foliación holomorfa de codimensión q sobre $U$.

La foliación $\mathcal{F}$ dada por la Proposición 2.3 , es tangente a la distribución de $(n-q)$-planos $\operatorname{Ker}(\Omega):=\cap_{j=1}^{q} \operatorname{Ker}\left(\Omega_{j}\right)$ y denotamos $T \mathcal{F}=\Omega$.

Proposición 2.4 Dos sistemas integrables de rangos máximos denotados por $\Omega=\left\{\Omega_{1}, \cdots, \Omega_{q}\right\}$ y $\Omega^{\prime}=\left\{\Omega_{1}^{\prime}, \cdots, \Omega_{q}^{\prime}\right\}$ definen la misma foliación en $U$ si, y solamente si, tenemos $\Omega_{i}^{\prime}=$ $\sum_{j=1}^{q} a_{i j} \Omega_{j}$ para algunas funciones holomorfas $a_{i j}$ en $U$, con la propiedad de que la matriz $A=\left(a_{i j}\right)_{i, j=1}^{q}$ es no singular en cada punto de $U$.

Las demostración de la Proposición 2.3 puede encontrarse en C. Camacho - A. Lins Neto ([3], pág. 175-189) y como consecuencia de la Proposición 2.3 tenemos la Proposición 2.4 donde la demostración puede encontrarse en L. Jurado ([3], pág. 28 ).

Dado el sistema $\Omega:=\left\{\Omega_{1}, \ldots, \Omega_{q}\right\}$, denotamos por $\mathcal{F}(\Omega)$ la foliación definida por el sistema.

Definición 2.4 Dadas las matrices $k \times l$ y $l \times s$ evaluadas de 1 -formas $A=\left(a_{i j}\right)$ e $B=(b)_{j t}$, respectivamente, podemos definir el produto cuña de $A$ y $B$ denotado por $A \wedge B$ como la matriz $k \times s$, tal que

$$
A \wedge B=\left(\sum_{j=1}^{l} a_{i j} \wedge b_{j t}\right)_{i, t} .
$$

De la misma manera, podemos definir la derivada exterior $d A$ como la matriz $k \times l$ matriz 2-formas cuando la entrada en la posición $(i, j)$ es la 2-forma $d a_{i j}$.

\section{Ejemplos de foliaciones}

Las definiciones y propiedades de formas diferenciables pueden encontrarse en M. Spivak ([9], pág. 201-227), los siguientes ejemplos que veremos de foliaciones de codimensión arbitraria por medio de formas diferenciables, son extensiones de los ejemplos de foliaciones de codimensión uno en A. Lins Neto (4], pág. 14). 
Ejemplo 3.1 Sean $\mathcal{F}_{1}, \ldots, \mathcal{F}_{q}$ foliaciones transversalmente afines de codimensión uno en $M$ que son transversales en todo $M$. Entonces la foliación intersección $\cap_{j=1}^{q} \mathcal{F}_{j}$ es una foliación de codimensión q en $M$ la cual es transversalmente afín.

En efecto, si $\mathcal{F}_{i}=\left\{\left(U_{j}^{i}\right), Y_{j}^{i}\right\}_{j=1}^{\infty}$ donde $Y_{j}^{i}: U_{j}^{i} \rightarrow \mathbb{C}$ es una submersión tal que

$$
Y_{j}^{i}=a_{j k}^{i} Y_{k}^{i}+b_{j k}^{i}, a_{j k}^{i}, b_{j k}^{i} \neq 0
$$

son constantes, entonces

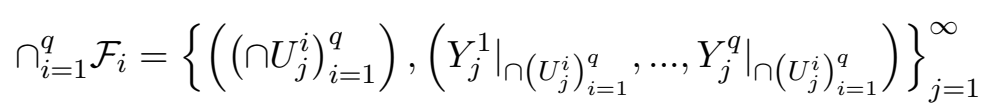

$\left(Y_{j}^{1}, \ldots, Y_{j}^{q}\right)$ es una submersión. Además, podemos escribir

$$
\left(\begin{array}{c}
Y_{j}^{1} \\
\vdots \\
Y_{j}^{q}
\end{array}\right)=\left(\begin{array}{cccc}
a_{j k}^{1} & 0 & \cdots & 0 \\
\vdots & a_{j k}^{1} & \cdots & 0 \\
\vdots & \vdots & \ddots & \vdots \\
0 & \cdots & 0 & a_{j k}^{q}
\end{array}\right)\left(\begin{array}{c}
Y_{k}^{1} \\
\vdots \\
Y_{k}^{q}
\end{array}\right)+\left(\begin{array}{c}
b_{j k}^{1} \\
\vdots \\
b_{j k}^{q}
\end{array}\right)
$$

donde $\quad\left(\begin{array}{cccc}a_{j k}^{1} & 0 & \cdots & 0 \\ \vdots & a_{j k}^{1} & \cdots & 0 \\ \vdots & \vdots & \ddots & \vdots \\ 0 & \cdots & 0 & a_{j k}^{q}\end{array}\right)$ es no singular, luego es una foliación transversalmente afín.

Observemos que de acuerdo con el Teorema 2.2, si $\mathcal{F}_{i}$ es una foliación transversalmente afín de codimensión uno. Entonces $F_{i}$ puede ser definida por una forma integrable $\Omega_{i}$, tal que existe $\eta_{i}$ y verifican $d \Omega_{i}=\eta_{i} \wedge \Omega_{i}$ y $d \eta_{i}=0$. Definimos $\Omega$ como la matriz $q \times 1$ cuyas componentes son $\Omega_{i}$ y $\eta$ una matriz $q \times q$ que tiene en su diagonal $\eta_{j}$ y demás componentes como ceros, $\Omega$ y $\eta$ evaluadas en $M$. En seguida, en la notación precedente tenemos $d \Omega=\eta \wedge \Omega$. Puesto que $\eta$ es diagonal, tenemos $d \eta=0=\eta \wedge \eta$.

Ejemplo 3.2 Sea $M$ una variedad compleja de dimensión $\geq 2$, y $\Omega=\left\{\omega_{1}, \ldots, \omega_{k}\right\}$ un sistema de 1 - formas cerradas holomorfas linealmente independientes en $M$, esto es

$$
d \Omega=\left(\begin{array}{c}
d \omega_{1} \\
d \omega_{2} \\
\vdots \\
d \omega_{k}
\end{array}\right)=\left(\begin{array}{c}
0 \\
0 \\
\vdots \\
0
\end{array}\right)
$$

Entonces, $\Omega$ es claramente integrable y por lo tanto define una foliación $\mathcal{F}$ en $M$. El Lema de Poincaré (ver [9], pág. 225) garantiza que dado un abierto simplemente conexo $U \subset M$, existe una aplicación holomorfa $f: U \rightarrow \mathbb{C}$, tal que $\left.\omega_{i}\right|_{U}=d f^{i}$. Además Si $g^{i}: V \rightarrow \mathbb{C}$ es una aplicación tal que $d g^{i}=\omega_{i}$, donde $U \cap V$ es conexo y no vacío, entonces $g^{i}$ y $f^{i}$ difieren por una constante en $U \cap V$. De esta forma, la foliación $\mathcal{F}$ puede ser localmente definida por funciones holomorfas en el siguiente sentido: existen colecciones $\mathcal{U}=\left\{U_{\alpha}\right\}_{\alpha \in A}, F=\left\{f_{\alpha}^{1}, \ldots, f_{\alpha}^{k}\right\}_{\alpha \in A}$, y $C=\left\{c_{\alpha \beta}^{1}, \ldots, c_{\alpha \beta}^{k}\right\}_{U_{\alpha} \cap U_{\beta}}$ tales que:

(a) $\mathcal{U}$ es una cobertura de $M$ por abiertos simplemente conexos. 
(b) Si $\alpha \in A$, entonces $f_{\alpha}^{i}$ es una aplicación holomorfa en $U_{\alpha} d f_{\alpha}^{i}=\left.\omega^{i}\right|_{U_{\alpha}}, 1 \leq i \leq k$.

(c) Si $U_{\alpha} \cap U_{\beta} \neq \emptyset$, entonces $U_{\alpha} \cap U_{\beta}$ es conexo, $c_{\alpha \beta} \in \mathbb{C}$ y $f_{\alpha}^{i}=f_{\beta}^{i}+c_{\alpha \beta}^{i}$ en $U_{\alpha \beta}$.

Así, $\mathcal{F}$ es una foliación regular donde se tiene

$$
\left(\begin{array}{c}
f_{\alpha}^{1} \\
f_{\alpha}^{2} \\
\vdots \\
f_{\alpha}^{k}
\end{array}\right)=\left(\begin{array}{cccc}
1 & 0 & \cdots & 0 \\
0 & 1 & \cdots & 0 \\
\vdots & \vdots & \ddots & \vdots \\
0 & 0 & \cdots & 1
\end{array}\right)\left(\begin{array}{c}
f_{\beta}^{1} \\
f_{\beta}^{2} \\
\vdots \\
f_{\beta}^{k}
\end{array}\right)+\left(\begin{array}{c}
c_{\alpha \beta}^{1} \\
c_{\alpha \beta}^{2} \\
\vdots \\
c_{\alpha \beta}^{k}
\end{array}\right)
$$

Entonces $\mathcal{F}$ es transversalmente afín. Por la relación $(3)$ diremos que $\mathcal{F}$ tiene una estructura transversal aditiva. En el caso en que sing $(\Omega) \neq \emptyset$, vemos que $\mathcal{F}$ tiene una estructura transversal aditiva en $M \backslash \operatorname{sing}(\mathcal{F})$.

Recíprocamente, si $\mathcal{F}$ es una foliación con estructura transversal aditiva en $M \backslash \operatorname{sing}(\mathcal{F})$ y tal que $\operatorname{cod}(\operatorname{sing}(\mathcal{F})) \geq 2$, entonces $\mathcal{F}$ puede ser definida por una 1 -forma holomorfa cerrada. En efecto, sean $\mathcal{U}=\left\{U_{\alpha}\right\}_{\alpha \in A}, F=\left\{f_{\alpha}^{1}, \ldots, f_{\alpha}^{k}\right\}_{\alpha \in A}, \mathrm{y} C=\left\{c_{\alpha \beta}^{1}, \ldots, c_{\alpha \beta}^{k}\right\}_{U_{\alpha} \cap U_{\beta}}$ colecciones que satisfacen $(a)$ y $(b)$, donde $\mathcal{F}$ es un cubrimiento abierto de $M \backslash \operatorname{sing}(\mathcal{F})$. De $(c)$ obtenemos que, si $U_{\alpha} \cap U_{\beta} \neq \emptyset$, entonces

$$
\left(\begin{array}{c}
d f_{\alpha}^{1} \\
d f_{\alpha}^{2} \\
\vdots \\
d f_{\alpha}^{k}
\end{array}\right)=\left(\begin{array}{c}
d f_{\beta}^{1} \\
d f_{\beta}^{2} \\
\vdots \\
d f_{\beta}^{k}
\end{array}\right) \text { en } U_{\alpha} \cap U_{\beta}
$$

Esto implica que existe un sistema de 1 -formas holomorfas $\Omega=\left\{\omega_{1}, \ldots, \omega_{k}\right\}$ en $M \backslash \operatorname{sing}(\mathcal{F})$ tal que $\left.\omega_{i}\right|_{U_{\alpha}}=d f_{\alpha}^{i}$, luego $\Omega$ es cerrada y define $\mathcal{F}$ en $M \backslash \operatorname{sing}(\mathcal{F})$. Por otro lado, como $\operatorname{cod}(\operatorname{sing}(\mathcal{F})) \geq 2$, el Teorema de Hartogs, ver ([5], pág. 228) implica que $\Omega$ se extiende a una forma holomorfa en $M$, la cual es también cerrada y define la foliación $\mathcal{F}$.

Podemos entonces enunciar el siguiente resultado:

Proposición 3.1 Sea $M$ una variedad holomorfa y $\mathcal{F}$ una foliación en $M$, cuyo conjunto singular tiene codimensión $\geq 2$. Entonces, $\mathcal{F}$ puede ser definida por una 1-forma cerrada si, y solamente si, $\mathcal{F}$ tiene una estructura transversal aditiva en $M \backslash \operatorname{sing}(\mathcal{F})$.

Definición 3.1 Sea $M$ una variedad compleja de dimensión $m$. Una foliación holomorfa singular de codimensión $q$ en $M$ definida por formas diferenciables es un objeto $\mathcal{F}$ dado por colecciones $\left\{\Omega_{\alpha}=\left\{\Omega_{\alpha}^{1}, \ldots, \Omega_{\alpha}^{q}\right\}\right\}_{\alpha \in A},\left\{U_{\alpha}\right\}_{\alpha \in A} y\left\{A_{\alpha \beta}\right\}_{U_{\alpha} \cap U_{\beta} \neq \phi}$ tales que:

1. $\left\{U_{\alpha}\right\}_{\alpha \in A}$ es un cubrimiento de $M$ por abiertos.

2. Para todo $\alpha, \Omega_{\alpha}$ es un sistema de 1-formas holomorfas integrable en $U_{\alpha}$ de rango máximo en cada punto.

3. Si $U_{\alpha} \cap U_{\beta} \neq \emptyset$, entonces $\Omega_{\alpha}=A_{\alpha \beta} \Omega_{\beta}$, donde $A_{\alpha \beta}$ es una matriz holomorfa en $U_{\alpha} \cap U_{\beta}$ no singular.

Ejemplo 3.3 Sean $N$ y $M$ variedades complejas y $\mathcal{F}$ una foliación de codimensión $k$ en $N$ como en la Definición 3.1. Dada una aplicación holomorfa no constante $f: M \rightarrow N$, podemos definir una foliación de codimensión $k$ en $M$, que será denotada por $f^{*}(\mathcal{F})$. 
En efecto, si $\mathcal{F}$ es definida por la terna $\left(\left(U_{j}\right)_{j \in J},\left(\Omega_{j}:=\left\{\omega_{1}^{j}, \ldots, \omega_{k}^{j}\right\}\right)_{j \in J},\left(A_{i j}\right)_{U_{i j}}\right)$, entonces $f^{*}(\mathcal{F})$ es definida por

$$
\left(\left(V_{j}\right)_{j \in J},\left(\Theta_{j}\right)_{j \in J},\left(B_{i j}\right)_{V_{i j} \neq \emptyset}\right)
$$

donde $V_{j}:=f^{-1}\left(U_{j}\right), \Theta_{j}:=f^{*}\left(\Omega_{j}\right)=\left\{f^{*}\left(\omega_{1}^{j}\right), f^{*}\left(\omega_{2}^{j}\right), \cdots, f^{*}\left(\omega_{k}^{j}\right)\right\}$ y $B_{i j}:=A_{i j} \circ f$.

Observe que $\left(\left(V_{j}\right)_{j \in J},\left(\Theta_{j}\right)_{j \in J},\left(B_{i j}\right)_{V_{i j} \neq \emptyset}\right)$ verifica

(a) $M=f^{-1}(N) \subset \bigcup f^{-1}\left(U_{j}\right)=\bigcup V_{j}$.

(b) $f^{*}\left(\omega_{1}^{j}\right) \wedge f^{*}\left(\omega_{2}^{j}\right) \wedge \ldots \wedge f^{*}\left(\omega_{k}^{j}\right) \wedge d\left(f^{*}\left(\omega_{i}\right)\right)=f^{*}\left(\omega_{1} \wedge \omega_{2} \wedge \ldots \wedge \omega_{k} \wedge d \omega_{j}\right)=0$.

(c) Como $\Omega_{j}=A_{i j} \Omega_{i}$ tenemos

$$
\left(\begin{array}{c}
\omega_{1}^{j} \\
\omega_{2}^{j} \\
\vdots \\
\omega_{q}^{j}
\end{array}\right)=\left(\begin{array}{cccc}
a_{11} & a_{12} & \cdots & a_{1 q} \\
a_{21} & a_{22} & \cdots & a_{2 q} \\
\vdots & \vdots & \ddots & \vdots \\
a_{q 1} & a_{q 2} & \cdots & a_{q q}
\end{array}\right)_{q \times q}\left(\begin{array}{c}
\omega_{1}^{i} \\
\omega_{2}^{i} \\
\vdots \\
\omega_{q}^{i}
\end{array}\right)
$$

luego

$$
f^{*}\left(\omega_{r}^{j}\right)=\sum_{s=1}^{n}\left(a_{r s} f\right) f^{*}\left(\omega_{s}^{i}\right)
$$

Por lo tanto, por la definición 3.1 tenemos que (a), (b) y (c) define una foliación en $f^{*}(\mathcal{F})$ que será llamada pull-back o pre-imagen de $\mathcal{F}$ por $f$.

\section{Primer resultado principal}

El Teorema a seguir prueba que una foliación de codimensión arbitaria en $\mathbb{C}^{n}$ genera una foliación de codimensión arbitraria en el espacio proyectivo complejo $\mathbb{C} \mathbb{P}^{n}$. Una prueba para una foliación de codimensión uno se puede consultar en A. Lins Neto - B. Scárdua ([5], pág 72).

Teorema 4.1 Sea $\mathcal{F}$ la foliación de codimensión q definida por un sistema integrable $\Omega$ en $\mathbb{C}^{n}$. Entonces podemos extender $\mathcal{F} a \mathbb{C P}^{n}$.

Demostración: Consideremos las cartas afines

$$
E_{j}:=\left\{[z]:=\left[z_{0}, \ldots, z_{j-1}, z_{j}, z_{j+1}, \ldots, z_{n}\right] \in \mathbb{C P}^{n} ; z_{j}=1\right\}
$$

Considere $\mathbb{C}^{n}$ como la carta afín $E_{0}$, el hiperplano del infinito de esa carta esta dado por $H=\left\{[z] \in \mathbb{C P}^{n} ; z_{0}=0\right\}$.

Considere el cambio de coordenadas entre $E_{1}$ y $E_{0}$.

$$
\phi(x)=\varphi_{0} \circ \varphi_{1}^{-1}\left(x_{1}, \ldots, x_{n}\right)=\varphi_{1}\left(1, x_{1}, \ldots, x_{n}\right)=\left(\frac{1}{x_{1}}, \frac{x_{2}}{x_{1}}, \ldots, \frac{x_{n}}{x_{1}}\right) .
$$

Por lo tanto, la expresión de $\Omega$ en la carta $E_{1}$ es el sistema de componentes

$$
\phi^{*}\left(\Omega^{i}\right)=f_{1}^{i}\left(\frac{1}{x_{1}}, \frac{x_{2}}{x_{1}}, \ldots, \frac{x_{n}}{x_{1}}\right) d\left(\frac{1}{x_{1}}\right)+\sum_{j=2}^{n} f_{j}^{i}\left(\frac{1}{x_{1}}, \frac{x_{2}}{x_{1}}, \ldots, \frac{x_{n}}{x_{1}}\right) d\left(\frac{x_{j}}{x_{1}}\right),
$$


donde $\Omega^{i}=\sum_{j=1}^{n} f_{j}^{i}(z) d z_{j} \not \equiv 0, \quad 1 \leq i \leq q$.

Sea

$$
f_{j}^{i}\left(z_{1}, \ldots, z_{n}\right)=f_{j}^{0 i}\left(z_{1}, \ldots, z_{n}\right)+\ldots+f_{j}^{r i}\left(z_{1}, \ldots, z_{n}\right)+\ldots
$$

tal que $f_{j}^{r i}(r \geq 0 \wedge 1 \leq j \leq n)$ son componentes homogéneas de grado $r$ de los $f_{j}^{i}$, respectivamente. Así

$$
\begin{aligned}
\phi^{*}\left(\Omega^{i}\right)= & \sum_{m=0}^{+\infty} f_{1}^{m i}\left(\frac{1}{x_{1}}, \frac{x_{2}}{x_{1}}, \ldots, \frac{x_{n}}{x_{1}}\right) \frac{-d x_{1}}{x_{1}^{2}}+\sum_{j=2}^{n} \sum_{m=0}^{+\infty} f_{j}^{m i}\left(\frac{1}{x_{1}}, \frac{x_{2}}{x_{1}}, \ldots, \frac{x_{n}}{x_{1}}\right) \frac{d x_{j} x_{1}-d x_{1} x_{j}}{x_{1}^{2}} \\
= & -\left(\sum_{m=0}^{+\infty} x_{1}^{-m} f_{1}^{m i}\left(1, x_{2}, \ldots, x_{n}\right) \frac{1}{x_{1}^{2}}+\sum_{j=2}^{n} \sum_{m=0}^{+\infty} x_{1}^{-m} f_{j}^{m i}\left(1, x_{2}, \ldots, x_{n}\right) \frac{x_{j}}{x_{1}^{2}}\right) d x_{1} \\
& +\sum_{j=2}^{n} \sum_{m=0}^{+\infty} x_{1}^{-m} f_{j}^{m i}\left(1, x_{1}, \ldots, x_{n}\right) \frac{d x_{j}}{x_{1}}
\end{aligned}
$$

luego

$$
\begin{aligned}
\phi^{*}\left(\Omega^{i}\right)= & \frac{1}{x_{1}^{k+2}}\left(-\sum_{m=0}^{+\infty} x_{1}^{k-m}\left(f_{1}^{m i}\left(1, x_{2}, \ldots, x_{n}\right)+\sum_{j=2}^{n} f_{j}^{m i}\left(1, x_{2}, \ldots, x_{n}\right) x_{j}\right) d x_{1}\right. \\
& \left.+\sum_{j=2}^{n} \sum_{m=0}^{+\infty} x_{1}^{k+1-m} f_{j}^{m i}\left(1, x_{2}, \ldots, x_{n}\right) d x_{j}\right) .
\end{aligned}
$$

Como los $f_{j / s}^{m i}$ son todos polinomios, la 1-forma descrita anteriormente es meromorfa, siendo $\left|\phi_{*}\left(\Omega^{i}\right)\right|_{\infty}=\left(x_{1}=0\right)$. Notamos que la multiplicidad de $x_{1}$ como polo de $\phi^{*}\left(\Omega^{i}\right)$ es $k_{i} \geq 2$. Podemos entonces escribir $\phi^{*}\left(\Omega^{i}\right)=\frac{\overline{\Omega^{i}}}{x_{1}^{k_{i}}}$, donde $\overline{\Omega^{i}}$ con coeficientes polinomiales.

Afirmación 4.1 El sistema de componentes integrables $\overline{\Omega^{i}}, 1 \leq i \leq q$, es integrable .

En efecto, como $\Omega_{1} \wedge \Omega_{2} \wedge \cdots \wedge \Omega_{q} \wedge d \Omega_{l}=0$ se tiene

$$
\phi^{*}\left(\Omega_{1}\right) \wedge \phi^{*}\left(\Omega_{2}\right) \wedge \cdots \wedge \phi^{*}\left(\Omega_{q}\right) \wedge d\left(\phi^{*}\left(\Omega_{l}\right)\right)=0 \mathrm{y} \phi^{*}\left(\Omega_{i}\right)=\frac{\overline{\Omega^{i}}}{x_{1}^{k_{i}}},
$$

entonces

$$
\frac{\overline{\Omega^{1}}}{x_{1}^{k_{1}}} \wedge \frac{\overline{\Omega^{2}}}{x_{1}^{k_{2}}} \wedge \cdots \wedge \frac{\overline{\Omega^{q}}}{x_{1}^{k_{q}}} \wedge\left(\frac{d \overline{\Omega^{l}}}{x_{1}^{k_{l}}}-\frac{k_{l} \overline{\Omega^{l}}}{x_{1}^{k_{l}+1}} \wedge d x_{1}\right)=0
$$

así

$$
\left(\frac{\overline{\Omega^{1}}}{x_{1}^{k_{1}}} \wedge \frac{\overline{\Omega^{2}}}{x_{1}^{k_{2}}} \wedge \cdots \wedge \frac{\overline{\Omega^{q}}}{x_{1}^{k_{q}}} \wedge \frac{d \overline{\Omega^{l}}}{x_{1}^{k_{l}}}\right)-\left(\frac{\overline{\Omega^{q}}}{x_{1}^{k_{1}}} \wedge \frac{\overline{\Omega^{2}}}{x_{1}^{k_{2}}} \wedge \cdots \wedge \frac{\overline{\Omega^{q}}}{x_{1}^{k_{q}}} \wedge \frac{k_{l} \overline{\Omega^{l}}}{x_{1}^{k_{l}+1}} d x_{1}\right)=0 .
$$

Por (4)

$$
\begin{gathered}
\overline{\Omega^{i}}=\left(-\sum_{m=0}^{+\infty} x_{1}^{k-m}\left(f_{1}^{m i}\left(1, x_{2}, \ldots, x_{n}\right)+\sum_{j=2}^{n} f_{j}^{m i}\left(1, x_{2}, \ldots, x_{n}\right) x_{j}\right) d x_{1}\right. \\
+\sum_{j=2}^{n} \sum_{m=0}^{+\infty} x_{1}^{k+1-m} f_{j}^{m i}\left(1, x_{2}, \ldots, x_{n}\right) d x_{j} .
\end{gathered}
$$


Se sigue que

$$
\begin{aligned}
& \frac{\overline{\Omega^{l}}}{x_{1}^{k_{l}}} \wedge \frac{k \overline{\Omega^{l}}}{x_{1}^{k_{l}+1}} d x_{1}=\left(\frac{1}{x_{1}^{k+2}}-\sum_{m=0}^{+\infty} x_{1}^{k-m}\left(f_{1}^{m l}\left(1, x_{2}, \ldots, x_{n}\right)+\sum_{j=2}^{n} f_{j}^{m l}\left(1, x_{2}, \ldots, x_{n}\right) x_{j}\right) d x_{1}\right. \\
& \left.+\frac{1}{x_{1}^{k+1}} \sum_{j=2}^{n} \sum_{m=0}^{+\infty} x_{1}^{k-m} f_{j}^{m l}\left(1, x_{2}, \ldots, x_{n}\right) d x_{j}\right) \wedge k \frac{1}{x_{1}^{k+2}} \sum_{j=2}^{n} \sum_{m=0}^{+\infty} x_{1}^{k-m} f_{j}^{m l}\left(1, x_{2}, \ldots, x_{n}\right) d x_{j} d x_{1} .
\end{aligned}
$$

Dado que $d x_{i} \wedge d x_{i}=0$ y $d x_{i} \wedge d x_{j}=0$ si $i \neq j$ tenemos $\frac{\overline{\Omega^{i}}}{x_{1}^{k_{i}}} \wedge \frac{\overline{\Omega^{2}}}{x_{1}^{k_{i}}} \wedge \cdots \wedge \frac{\overline{\Omega^{q}}}{x_{1}^{k_{i}}} \wedge \frac{k \overline{\Omega^{l}}}{x_{1}^{k+1}} d x_{1}=0$, luego el sistema de componentes $\overline{\Omega^{i}}$ es integrable, es decir:

$$
\overline{\Omega^{1}} \wedge \overline{\Omega^{2}} \wedge \cdots \wedge \overline{\Omega^{q}} \wedge d \overline{\Omega^{l}}=0 .
$$

Observemos por los cálculos hechos que el orden del polo $k_{i}$ es igual en todas las cartas $E_{j}$ para $\Omega^{i}$.

Podemos entonces, definir una foliación $\overline{\mathcal{F}}$ en $\mathbb{C P}^{n}$ tal que $\overline{\mathcal{F}}$ restringida a $E^{j}$ es definida por el sistema de 1 -formas $Z^{j}$, esto es:

(a) $Z^{0}=\Omega=\left\{\Omega^{1}, \ldots, \Omega^{q}\right\}$ en $E_{0}$.

(b) $Z^{1}=\left\{\left(\varphi_{0} \circ \varphi_{1}^{-1}\right)^{*}\left(\Omega^{1}\right) x_{1}^{k_{1}}, \ldots,\left(\varphi_{0} \circ \varphi_{1}^{-1}\right)^{*}\left(\Omega^{q}\right) x_{1}^{k_{q}}\right\}$ en $E_{1}$.

(c) $Z^{j}=\left\{\left(\varphi_{0} \circ \varphi_{l}^{-1}\right)^{*}\left(\Omega^{1}\right) x_{j}^{k_{1}}, \ldots,\left(\varphi_{0} \circ \varphi_{l}^{-1}\right)^{*}\left(\Omega^{q}\right) x_{j}^{k_{q}}\right\}$ en $E_{j}$.

Así, según la definición 3.1, tenemos una foliación en $\mathbb{C P}^{n}$ :

1. $\cup_{\alpha=0}^{n} E_{\alpha}=\mathbb{C P}^{n}$.

2. El sistema de componentes integrables $\overline{\Omega^{i}}$ es integrable .

3. En $E_{1} \cap E_{0}$ se verifica la siguiente igualdad

$$
\left(\begin{array}{cccc}
x_{1}^{k_{1}} & 0 & \cdots & 0 \\
0 & x_{1}^{k_{2}} & \cdots & 0 \\
\vdots & \vdots & \ddots & \vdots \\
0 & 0 & \cdots & x_{1}^{k_{q}}
\end{array}\right)\left(\begin{array}{c}
\left(\varphi_{1}^{-1}\right)^{*} \Omega^{1} \\
\left(\varphi_{1}^{-1}\right)^{*} \Omega^{2} \\
\vdots \\
\left(\varphi_{1}^{-1}\right)^{*} \Omega^{q}
\end{array}\right)=\left(\begin{array}{c}
\left(\varphi_{0}^{-1}\right)^{*}\left(Z^{1}\right)^{1} \\
\left(\varphi_{0}^{-1}\right)^{*}\left(Z^{1}\right)^{2} \\
\vdots \\
\left(\varphi_{0}^{-1}\right)^{*}\left(Z^{1}\right)^{q}
\end{array}\right)
$$

De manera análoga en $E_{i} \cap E_{j}$. La foliación $\overline{\mathcal{F}}$ obtenida es llamada compactificación de $\mathcal{F}$.

\section{Segundo Resultado principal}

A seguir, un teorema que caracteriza una foliación transversalmente afín de codimensión $q$, análogo al que sucede en foliaciones transversalmente afínes de codimensión uno. Para más detalles y la recíproca del Teorema 5.1 se puede consultar en B. Scárdua ([6], pág. 3-8). 
Teorema 5.1 Sea $\mathcal{F}$ una foliación holomorfa de codimensión $q$ en $M$. Si la foliación $\mathcal{F}$ es transversalmente afín en $M$, entonces existe un cubrimiento abierto $\cup_{i \in I} U_{i}=M$ y matrices holomorfas $q \times 1, q \times q$ de 1 -formas, $\Omega_{i}$, $\eta_{i}$ en $U_{i} \forall i \in I$, de manera que:

1. $\left.\mathcal{F}\right|_{U_{i}}=\mathcal{F}\left(\Omega_{i}\right)$.

2. $d \Omega_{i}=\eta_{i} \wedge \Omega_{i}$ y $d \eta_{i}=\eta_{i} \wedge \eta_{i}$.

3. Si $U_{i} \cap U_{j} \neq \emptyset$, entonces tenemos $\Omega_{i}=G_{i j} \cdot \Omega_{j}$ y $\eta_{i}=\eta_{j}+d G_{i j} \cdot G_{i j}^{-1}$ para alguna aplicación holomorfa $G_{i j}: U_{i} \cap U_{j} \rightarrow G L_{q}(\mathbb{C})$.

Además, dos de estas colecciones $\left(\Omega_{i}, \eta_{i}, U_{i}\right)_{i \in I}$ y $\left(\Omega_{i}^{\prime}, \eta_{i}^{\prime}, U_{i}^{\prime}\right)_{i \in I}$ definen la misma estructura transversal afín de $\mathcal{F}$, si, y solamente si, tenemos $\Omega_{i}^{\prime}=G_{i} \cdot \Omega_{i}$ e $\eta_{i}^{\prime}=\eta_{i}+d G_{i} \cdot G_{i}^{\prime}$ para alguna aplicación holomorfa $G_{i}: U_{i} \rightarrow G L_{q}(\mathbb{C})$.

Enunciamos algunos resultados que serán utilizados en la prueba del Teorema 5.1. Iniciamos con el siguiente lema bien conocido de análisis real, adaptado al caso holomorfo:

Lema 5.2 Sea $X: U \subset \mathbb{C}^{n} \rightarrow G L_{q}\left(\mathbb{C}^{n}\right)$ una aplicación holomorfa, entonces

$$
d\left(X^{-1}\right)=-X^{-1} \cdot d X \cdot X^{-1} .
$$

Demostración: Como $d(X)(z)(v)=v(X), z \in \mathbb{C}^{n}, v \in \mathbb{C}^{n}$, donde $v=\sum_{i=1}^{n} a_{i} \frac{\partial}{\partial z_{i}}$, así

$$
d X(z)(v)=\sum_{i=1}^{n} a_{i}(z) \frac{\partial X}{\partial z_{i}}(z) .
$$

Por otro lado

$$
\frac{\partial\left(X X^{-1}\right)}{\partial y_{j}}=\frac{\partial I_{n}}{\partial y_{j}}=0
$$

y

$$
\frac{\partial\left(X X^{-1}\right)}{\partial y_{j}}=\frac{\partial X}{\partial y_{j}} \cdot X^{-1}+X \frac{\partial X^{-1}}{\partial y_{j}}
$$

de (6) y (7), tenemos

$$
\frac{\partial X^{-1}}{\partial y_{i}}=-X^{-1} \frac{\partial X}{\partial y_{j}} X^{-1}
$$

entonces por (8)

$$
\begin{aligned}
d X^{-1}(w)(v)=v\left(X^{-1}\right) & =\sum_{j=1}^{m} b j \frac{\partial}{\partial y_{j}} X^{-1} \\
& =X^{-1} \cdot \sum_{j=1}^{m} b_{j} \frac{\partial X}{\partial y_{j}} \cdot X^{-1}
\end{aligned}
$$

Por lo tanto

$$
d\left(X^{-1}\right)=-X^{-1} \cdot d X \cdot X^{-1} .
$$


Lema 5.3 Sean $X: U \subset \mathbb{C}^{n} \rightarrow G L_{q}(\mathbb{C})$ una aplicación holomorfa y $\eta$ es definida diagonal $\eta=d X \cdot X^{-1}$, entonces tenemos $d \eta=\eta \wedge \eta$.

Demostración: Por el Lema 5.2 tenemos

$$
d\left(X^{-1}\right)=-X^{-1} \cdot d X \cdot X^{-1} .
$$

Así

$$
\begin{aligned}
d \eta=d\left(d X \cdot X^{-1}\right) & =d(d X) \wedge X^{-1}+(-1) d X \wedge d\left(X^{-1}\right) \\
& =(-1) d X \wedge\left(-X^{-1} \cdot d X \cdot X^{-1}\right) \\
& =\left(d X \cdot X^{-1}\right) \wedge\left(d X \cdot X^{-1}\right) \\
& =\eta \wedge \eta
\end{aligned}
$$

Lema 5.4 Dado $\eta$ una matriz holomorfa $q \times q$ de 1-formas en $U \subset \mathbb{C}^{n}$ tal que $d \eta=\eta \wedge \eta, y$ una aplicación holomorfa $G: U \rightarrow G L_{q}(\mathbb{C})$, entonces la 1 -forma $\widetilde{\eta}:=\eta+d G \cdot G^{-1}$ satisface $d \widetilde{\eta}=\widetilde{\eta} \wedge \widetilde{\eta}$

Demostración: Observe que

$$
\begin{aligned}
d \widetilde{\eta} & =d \eta+d\left(d G \cdot G^{-1}\right) \\
& =\eta \wedge \eta+d G \cdot G^{-1} \wedge d G \cdot G^{-1}
\end{aligned}
$$

Por otro lado

$$
\begin{aligned}
\widetilde{\eta} \wedge \widetilde{\eta} & =\left(\eta+d G \cdot G^{-1}\right) \wedge\left(\eta+d G \cdot G^{-1}\right) \\
& =\eta \wedge \eta+\eta \wedge d G \cdot G^{-1}+d G \cdot G^{-1} \wedge \eta+d G \cdot G^{-1} \wedge \eta+d G \cdot G^{-1} \wedge d G \cdot G^{-1} \\
& =\eta \wedge \eta+d G \cdot G^{-1} \wedge d G \cdot G^{-1} .
\end{aligned}
$$

Por lo tanto, $d \widetilde{\eta}=\widetilde{\eta} \wedge \widetilde{\eta}$.

Lema 5.5 Sean $G, G^{\prime}: U \subset \mathbb{C}^{n} \rightarrow G L_{q}(\mathbb{C})$ aplicaciones holomorfas. Entonces tenemos $d G \cdot G^{-1}=d G^{\prime} \cdot G^{\prime-1}$ si, y solamente si, $G^{\prime}=G \cdot A$ para alguna constante $A: U \rightarrow G L_{q}(\mathbb{C})$.

Demostración: Primero asumimos que $G^{\prime}=G \cdot A$ con $A$ localmente constante. Así tenemos $G^{-1} \cdot G^{\prime}=A$ y por lo tanto $d\left(G^{-1} \cdot G^{\prime}\right)=d A=0$ en $U$, esto implica $d\left(G^{-1}\right) \cdot G^{\prime}+G^{-1} \cdot d\left(G^{\prime}\right)=0$. Usando que $d\left(G^{-1}\right)=-G^{-1} \cdot d G \cdot G^{-1}$ tenemos

$$
-G^{-1} \cdot d G \cdot G^{-1}+G^{-1} \cdot d G^{\prime}=0 .
$$

Multiplicando a la izquierda por G obtenemos:

$$
-d G \cdot G^{-1} \cdot G^{\prime}+d G^{\prime}=0 .
$$

Multiplicando a la derecha esta última igualdad por $G^{\prime-1}$ obtenemos

$$
-d G \cdot G^{-1}+d G^{\prime} \cdot G^{\prime-1}=0
$$

lo que comprueba la primera parte.

Ahora supongamos que $d G \cdot G^{-1}=d G^{\prime} \cdot G^{\prime-1}$ en $U$. Definimos $A=G^{-1} \cdot G^{\prime}$, así tenemos que $G^{\prime}=G \cdot A$. Basta demostrar que $d A=0$ en $U$.

En efecto,

$$
d A=d\left(G^{-1} \cdot G^{\prime}\right)=d G^{-1} \cdot G^{\prime}+G^{-1} \cdot d G^{\prime} .
$$


Puesto que

$$
d G^{-1}=-G^{-1} \cdot d G \cdot G^{-1}
$$

obtenemos

$$
\begin{aligned}
d A & =-G^{-1} \cdot d G \cdot G^{-1} \cdot G^{\prime}+G^{-1} \cdot d G^{\prime} \\
& =-G^{-1} \cdot\left(d G \cdot G^{-1}-d G^{\prime} \cdot G^{\prime}-1\right) \cdot G^{\prime} .
\end{aligned}
$$

Por hipótesis $d G \cdot G^{-1}=d G^{\prime} \cdot G^{\prime}-1$, luego $d A=0$, concluyendo así la prueba.

Proposición 5.6 Sea $\mathcal{F}$ una foliación holomorfa de codimensión q sobre $M$. Supongamos que $\mathcal{F}$ es definida por algún sistema holomorfo integrable $\left\{\Omega_{1}, \ldots, \Omega_{q}\right\}$ de 1-formas. Si $\mathcal{F}$ es transversalmente afin, entonces existe una matriz holomorfa $q \times q$ de 1 -formas $\eta=\left(\eta_{i j}\right)$ que satisface:

$$
d \Omega=\eta \wedge \Omega, \quad d \eta=\eta \wedge \eta \quad \text { onde } \quad \Omega=\left(\begin{array}{c}
\Omega_{1} \\
\vdots \\
\Omega_{q}
\end{array}\right)
$$

Demostración: Sea $\left\{\Omega_{1}, \ldots, \Omega_{q}\right\}$ un sistema holomorfo integrable, el cual define $\mathcal{F}$ en $M$ y supongamos que $\left\{Y_{i}: U_{i} \rightarrow \mathbb{C}^{q}\right\}_{i \in I}$ es una estructura afín transversal para $\mathcal{F}$ en $M$, con

$$
Y_{i}=A_{i j} Y_{j}+B_{i j} \text { en } U_{i} \cap U_{j} \neq \emptyset
$$

como en la definición 2.2.

Ya que las submersiones $Y_{i}$ también definen $\mathcal{F}$, por la proposición 2.4 podemos escribir

$$
\left(\begin{array}{c}
\Omega_{1} \\
\vdots \\
\Omega_{q}
\end{array}\right)=\Omega=G_{i} \cdot d Y_{i}=G_{i} \cdot\left(\begin{array}{c}
d Y_{i}^{1} \\
d Y_{i}^{2} \\
\ldots \\
d Y_{i}^{q}
\end{array}\right)
$$

en cada $U_{i}$, para alguna aplicación holomorfa $G_{i}: U_{i} \rightarrow G L_{q}(\mathbb{C})$.

En cada $U_{i} \cap U_{j} \neq \emptyset$ tenemos

$$
G_{i} \cdot d Y_{i}=G_{j} \cdot d Y_{j}
$$

y sigue de (9) que

$$
G_{j}=G_{i} \cdot A_{i j}
$$

De acuerdo al Lema 5.5 , sigue inmediatamente

$$
d G_{j} \cdot G_{j}^{-1}=d G_{i} \cdot G_{i}^{-1}, \quad \text { en } \quad U_{i} \cap U_{j} \neq \emptyset .
$$

Esto permite definir $\eta$ en $M$ por

$$
\left.\eta\right|_{U_{i}}=d G_{i} \cdot G_{i}^{-1}
$$

De acuerdo al Lema 5.4 tenemos $d \eta=\eta \wedge \eta$. También, tenemos en cada $U_{i}$

$$
\begin{aligned}
d \Omega=d\left(G_{i} \cdot d Y_{i}\right) & =d G_{i} \wedge d Y_{i} \\
& =d G_{i} \cdot G_{i}^{-1} \wedge d Y_{i} \\
& =d G_{i} \cdot G_{i}^{-1} \wedge G_{i} \cdot d Y_{i} \\
& =\eta \wedge \Omega .
\end{aligned}
$$

Por lo tanto, el par $(\Omega, \eta)$ satisface $d \Omega=\eta \wedge \Omega$ y se concluye la prueba. 
Demostración del Teorema 5.1 La Proposición 5.6 muestra que si $\mathcal{F}$ es transversalmente afín en $M$, entonces podemos construir una colección $\left(\Omega_{j}, \eta_{j}\right)$ en los subconjuntos abiertos $U_{j} \subset M$ que cubre $M$, como lo establecido.

Finalmente, suponga que $(\Omega, \eta)$ y $\left(\Omega^{\prime}, \eta^{\prime}\right)$ definen la misma estructura transversal para $\mathcal{F}$ en $U_{i}$. Como $\Omega$ y $\Omega^{\prime}$ definen $\mathcal{F}$, tenemos $\Omega^{\prime}=G \cdot \Omega$. Usando la misma notación de siempre, escribimos localmente $\Omega=G_{i} \cdot d Y_{i}, \Omega^{\prime}=G_{i}^{\prime} \cdot d Y_{i}, \eta=\frac{d G_{i}}{G_{i}}$ y $\eta^{\prime}=\frac{d G_{i}^{\prime}}{G_{i}^{\prime}} \sin$ embargo $G_{i}^{\prime}=G \cdot G_{i}$, así $\eta^{\prime}=\eta+\frac{d G}{G}$, completando la prueba del Teorema 5.1 .

\section{Conclusión}

Los resultados aquí presentados nos muestran la relación entra las foliaciones y las formas diferenciables. El primer resultado nos dice en forma general que una foliación de codimensión arbitraria induce una foliación en el espacio proyectivo. El segundo resultado nos muestra que una foliación transversalemnte afín se puede caracterizar matricialmente con entradas que son formas diferenciables. La recíproca es verdadera, pero para probarla es preciso definir conceptos avanzados que pueden encontrarse en C. Godbillon ([2], pág. 139-163) que se pretenden presentar en un próximo trabajo.

\section{Referencias bibliográficas}

[1] Camacho, C. y Neto, L. (1979). Teoria geométrica das folheações. Rio de Janeiro, Brasil: Projeto Euclides, IMPA.

[2] Godbillon, C. (1991). Feuilletages. Études Géométriques. Progress in Mathematics (Libro 98). Birkhauser Verlag, Basel.

[3] Jurado, L. (2017), Sobre folheações complexas com estrutura transversalmente afins em codimensão arbitrária (Tese de doutorado). Instituto de Matemática-UFRJ, Río de Janeiro, Brasil.

[4] Lins-Neto, L. (2007). Componentes irredutiveis dos espações de folheações. 26 Coloquio Brasileiro de Matemática.

[5] Scárdua, B. y Lins-Neto, A. (1997). Introdução á Teoria das Folheações Algebraicas Complexas, 21 Coloquio Brasileiro de Matemática.

[6] Scárdua, B. (2014). On Elementary Integration and Affine Transverse Structures for Algebraic Foliations of Arbitrary codimensión. Arxiv:1411.0262,

[7] Scárdua, B. (2011). Differential Algebra and Liouvillian first integrals of foliations. Journal of Pure and Applied Algebra, 215(5), 764-788.

[8] Singer, M. (1992). Liouvillian first integral of differential equations. The Transactions of the American Mathematical Society, 333(2), 673-688.

[9] Spivak, M. (1979). A Comprehensive Introduction to Differential Geometry (Vol. 1 second edition). Berkeley, States United: Publish or Perish. 